\title{
Structural flexibility and functional interaction of Mediator Cdk8 module
}

\author{
Xuejuan Wang ${ }^{1^{*}}$, Jianye Wang ${ }^{1^{*}}$, Zhenrui Ding ${ }^{1}$, Jinhua $\mathrm{Ji}^{1}$, Qianqian Sun ${ }^{1}$, Gang Cai ${ }^{1,2} \bowtie$ \\ ${ }^{1}$ School of Life Sciences, University of Science and Technology of China, Hefei 230026, China \\ ${ }^{2}$ Hefei National Laboratory for Physical Sciences at the Microscale, Center for Integrative Imaging, Hefei 230026, China \\ $\square$ Correspondence: gcai@ustc.edu.cn
}

Received August 2, 2013 Accepted August 26, 2013

\begin{abstract}
Mediator is a highly conserved large protein complex $(25$ proteins, $>1000 \mathrm{kDa}$ ) and preeminently responsible for eukaryotic transcription, which contains a dissociable 'Cdk8 module'. Although increasing evidence demonstrates that Cdk8 module plays both positive and negative roles in transcription regulation, the detailed structure, and subunit organization, molecular mechanism how it regulates transcription remain elusive. Here we used single-particle electron microscopy to characterize the structure and subunit organization of the Cdk8 module and illuminated the substantial mobility of the Med13 subunit results in the structural flexibility. The Cdk8 module interaction with core Mediator is concurrent with active transcription in vivo. An interaction with the Cdk8 module induces core Mediator into very extended conformation in vitro, which is presumed to be an active functional state of Mediator. Taken together, our results illuminated the detailed architecture of Cdk8 module, and suggested the Cdk8 module could positively regulate transcription by modulating Mediator conformation.
\end{abstract}

KEYWORDS Mediator, Cdk8 kinase module, transcription regulation

\section{INTRODUCTION}

Regulation of eukaryotic transcription is largely focused on the transcription initiation process, which involves assembly of the large pre-initiation complex (PIC). Typically the PIC is composed of RNA polymerase II (Pol II) and six general transcription factors (GTFs) including IIA, IIB, IID, IIE, IIF, and IIH (Lee and Young, 2000). Transcription factors (including activators and repressors) control the transcription initiation and

*These authors contributed equally to the work.

(c) Higher Education Press and Springer-Verlag Berlin Heidelberg 2013 efficiency by interacting with regulatory DNA. Mediator, a large protein complex (>1000 kDa) comprising 25 different proteins, is preeminently responsible for PIC assembly and conveying regulatory signals from DNA-binding transcription factors to the PIC (Malik and Roeder, 2000; Naar et al., 2001; Kornberg, 2005). Mediator is organized structurally and functionally in three core modules (Head, Middle and Tail module) and a dissociable 'Cdk8 module' consisting of the cyclin-dependent kinase Cdk8, its partner CycC, Med12, and Med13 (Borggrefe et al., 2002; Chadick and Asturias, 2005). Core Mediator contains three stable modules: Head, Middle, and Tail, while the dissociable Cdk8 module dynamically interacts with core Mediator.

Understanding both the structure and regulation of the Mediator complex is essential to elucidate the mechanism of gene regulation (Chadick and Asturias, 2005; Lariviere et al., 2012). Our understanding of Mediator assembly and its role in regulating transcription has been impeded so far by the large size, low abundance, dynamic composition, and intrinsic flexibility of Mediator, which has been a formidable challenge for structural analysis. Single particle electron microscopy (EM) is uniquely suited for structural analysis of the large multi-protein complexes and previous EM studies have contributed significantly to understanding functional architecture of Mediator (Asturias et al., 1999; Dotson et al., 2000; Taatjes et al., 2002; Cai et al., 2009). The most detailed cryo-EM structure of yeast core Mediator highlights the remarkable conformational flexibility and reveals its modularity features (Head, Middle, Tail, and Arm) (Cai et al., 2009). Not only acquiring the structural information, single particle EM is ideally suited to characterize the conformation of Mediator under different conditions and illuminate how it is affected by interaction with various factors. Understanding the interaction of Mediator with the general RNAPII machinery has derived from EM structures of the MediatorRNAPII complex (Davis et al., 2002; Bernecky et al., 2011), Mediator-TFIIH complex (Lee et al., 2010), Mediator Head- 
Yeast whole cell extraction

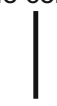

Ammonium sulfate cut $(30 \%-55 \%)$
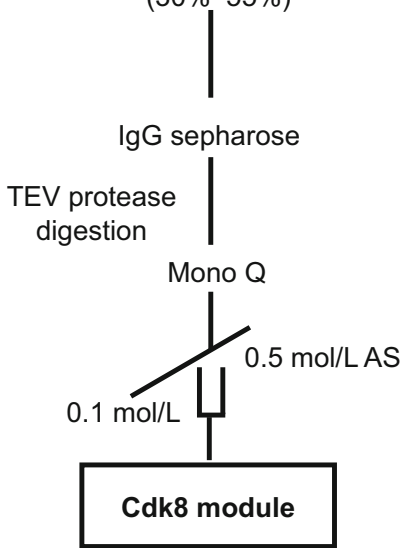

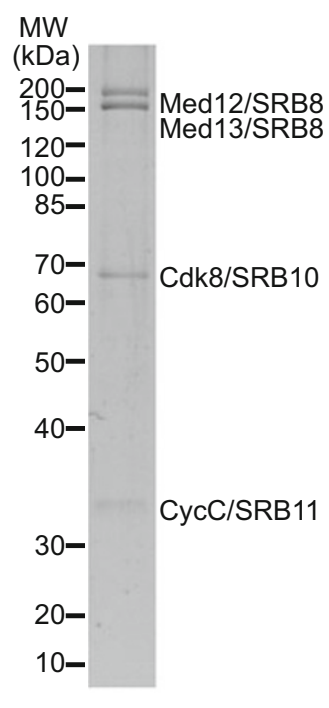

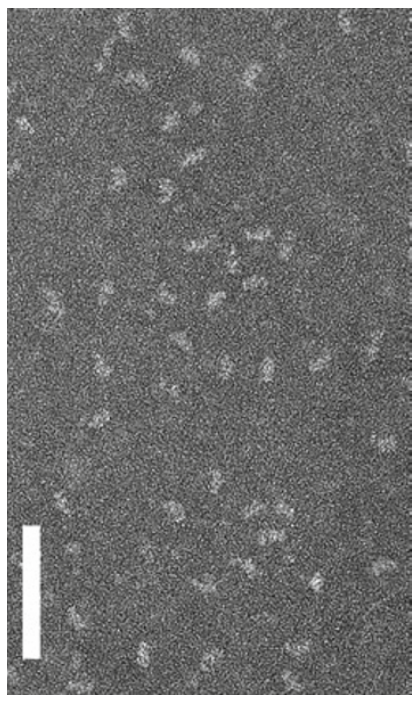

Figure 1. Preparation and EM characterization of the Mediator Cdk8 module. (A) Scheme for affinity purification of Mediator Cdk8 module from the whole cell extraction of tagged S. cerevisiae cells. (B) Ruby-stain SDS-PAGE analysis of purified yeast Cdk8 module. (C) A micrograph showing single Cdk8 module particles preserved in uranyl formate. Scale bar, $100 \mathrm{~nm}$.
TBP (Cai et al., 2010), and Mediator Head-RNAPII (Cai et al., 2010, 2012).

The cyclin-dependent kinase Cdk8, its partner CycC, Med12, and Med13 form a dissociable 'Cdk8 module', which is critical for transcriptional regulation. Notably, Cdk8, a component of Mediator, is an important oncogene (Firestein et al., 2008; Adler et al., 2012). Two previous publications on EM structural studies on Cdk8 module from fission yeast and human have reported it interact differently with Mediator in the two species and could repress transcription through either occupying the RNAPII binding sites or locking the conformation of Mediator to prevent RNAPII binding, respectively (Elmlund et al., 2006; Knuesel et al., 2009a). However, the detailed structure and subunit organization of Cdk8 module, the precise mode of interaction between Cdk8 module and Mediator, and the mechanism of regulation by Cdk8 module remain unclear. Moreover, instead of the established repressive role in transcription (Holstege et al., 1998; Myers et al., 1998; Taatjes et al., 2002), the Cdk8 module has been increasingly substantiated that could also contribute to transcription activation (Belakavadi and Fondell, 2010; Donner et al., 2010; Conaway and Conaway, 2011). Therefore, we are interested to study structure, subunit organization, and functions of Cdk8 module in more detail to achieve a comprehensive understanding of the Cdk8 module's function in transcription regulation.

We used single-particle electron microscopy, along with biochemical assays to characterize the structure and subunit organization of the Cdk8 module from Saccharomyces cerevisiae (S. cerevisiae). When we are preparing the manuscript, the structure of the yeast Cdk8 module has been published online (Tsai et al., 2013) and some of the data of the subunit organization of Cdk8 module presented in this paper are consistent with our findings. However, our EM structure, for the first time, revealed the Cdk8 module adopts several different conformations corresponding to the mobility of the Med13 subunit, which appears to harbor important functional implications. Moreover, we characterized, by structural and biochemical means, the interactions of Mediator with the Cdk8 module and RNAPII. Surprisingly, Mediator harbors strong interaction with RNAPII during transcription inactive stationary phase and only minor binding with RNAPII in transcription highly active exponential phase. In contrast, Cdk8 module prefers to strongly interact with Mediator during exponential phase, which is concurrent with active transcription, thus suggesting Cdk8 module is involved in transcription activation. In vitro, both purified RNAPII and Cdk8 module could dynamically bind to the Mediator independently of other factors. The substantial conformational changes of Mediator upon interaction with RNAPII and Cdk8 module is comparable and Mediator was induced to adopt extended conformation where Middle and Tail module move far apart. These results shed light on the regulation of transcription by Cdk8 module and suggest how Cdk8 could influence transcription by modulating Mediator conformation.

\section{RESULTS AND DISCUSSION}

\section{Purification of the Mediator Cdk8 module from S. cerevisiae}

Mediator is a low abundance, fragile, and large protein complex (>1000 kDa) (In S. cerevisiae, comprising 25 different proteins), which posed a serious impediment for biochemical preparation. We have established a novel affinity purification method (Fig. 1A) that readily and reproducibly yields highly homogeneous and functional yeast core Mediator complex (dissociable Cdk8 module is absent), which has resulted in the first cryo-EM reconstruction of Mediator (Cai et al., 2009). Moreover, we also successfully applied the method to purify the Cdk8 module (Fig. 1B). To further remove some minor contaminations, an ion exchange Mono $\mathrm{Q}$ column is employed to polish the purification. The improved procedures yielded the complete 


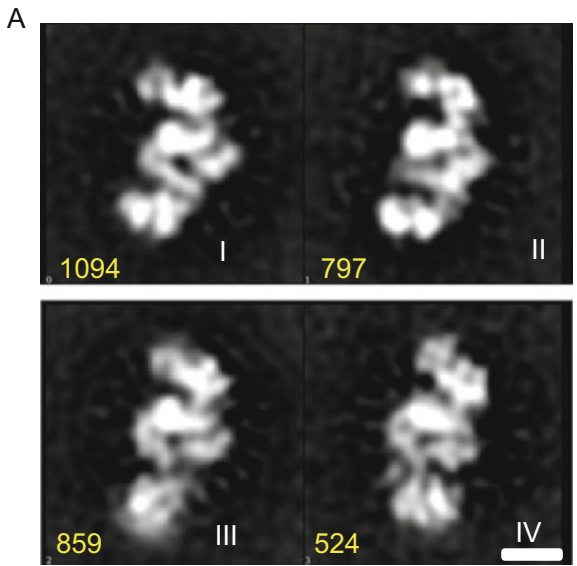

C
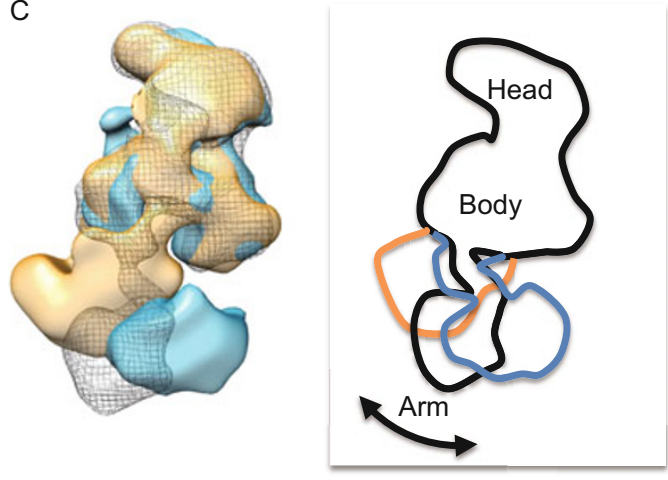

B
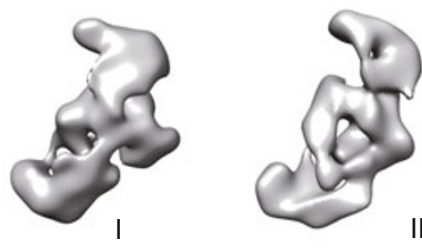

Front

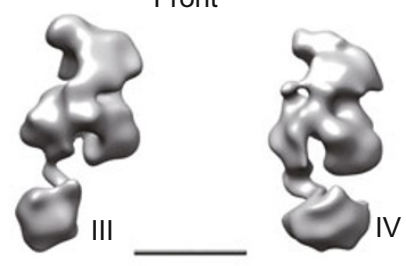

I

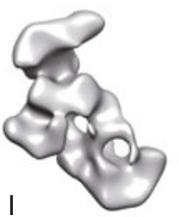

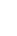

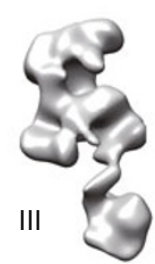

II

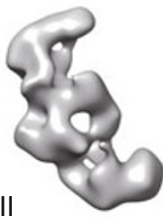

Back

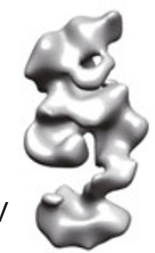

Figure 2. Structural flexibility of Cdk8 module. (A) Four different conformations of the Cdk8 module were identified through reference-free alignment and classification of EM images of particles preserved in stain. The number of images included in each class average is indicated under each average. Cdk8 module particles were not evenly distributed among the four different conformations that differ mainly in the position of one protruding arm domain. Information from images of tilted particles was used to obtain $3 \mathrm{D}$ reconstruction of the Cdk8 module in all four different conformations. Scale bar, $100 \AA$. (B) Front and back views of 3D RCT reconstructions of Cdk8 module corresponding to the four conformations. Scale bar, $100 \AA$. (C) Superposition of Cdk8 module conformer I (yellow), conformer IV (blue), and conformer III (gray) structures shows the structural flexibility exists mainly in the "Leg" domain. The arrows indicate the direction of movement in the proposed conformational change. 4-subunit, 427-kDa Cdk8 module purification, which was uniform in composition, with equimolar amount of subunits, on the basis of SDS/PAGE analysis (Fig. 1B). The quality of the Cdk8 module purification was assessed by direct examination of the homogeneity and structural integrity of the particles by EM. We imaged Cdk8 module particles in the electron microscope after preservation in stain. The particles seemed well preserved and similar in size and overall shape (Fig. 1C).

\section{Structural analysis of the Cdk8 module using single-particle EM}

Upon adsorption to amorphous carbon support films, all Cdk8 complexes showed strongly preferred orientation; so image alignment and classification could be used to distinguish differences in molecular conformation, without having to simultaneously address differences in orientations. Reference-free alignment and averaging of Cdk8 module particle images generated two-dimensional (2D) maps showed " $\xi$ "-shaped architecture with a central core lobe embraced by two flanked arms (Fig. 2A). Image classification revealed structural variability resulting from large changes in the position of one of the two protruding arms at the distal ends of the Cdk8 module structure. Particles adopted four distinct conformations that differed in the position of the protruding arm domain (termed with "Leg"), which is highly mobile and flexibly connected to the central lobe, and only ordered in conformer I of Cdk8 module (Fig. 2C).
We obtained three-dimensional (3D) structures of each Cdk8 module conformation (Figs. $2 \mathrm{~B}$ and $\mathrm{S} 1$ ) from images of tilted stained particles using the random conical tilt (RCT) method (Radermacher, 1988). Simply, pairs of images of negatively stained particles were recorded at tilt angles of 0 and -55 degrees, so 3D structures could be calculated by the RCT method. The resolution of the reconstructions was estimated to be about $30-35 \AA$ by the Fourier shell correlation method. We attempted cryo-EM analysis, but the conformational flexibility makes the Cdk8 module a very challenging target for reliable $3 \mathrm{D}$ reconstructions. The structural variability evident in projection maps was clearly reflected in the 3D structures. Especially, the "Leg" domain is highly mobile and flexibly connected, which constitute the major structural flexibility of the Cdk8 module (Fig. 2C).

Through more careful biochemistry and computationally sorting of different conformational states of the Cdk8 module, we acquired well-defined sub-populations for the $3 \mathrm{D}$ reconstructions and firstly delineated the substantial mobility of the "Leg" domain of the Cdk8 module. In contrast, Tsai et al. did not sort the inherent conformational heterogeneity of the Cdk8 module and reconstruct only one conformation of the 3D structure of Cdk8 module, which is corresponding to the conformer II of our reconstructions (Tsai et al., 2013). Interestingly, the 3D structure of the Cdk8 module looks strikingly similar to that of human CDK8 module (Knuesel et al., 2009a), especially the high mobile "Leg" domain appears to be nicely corresponding 


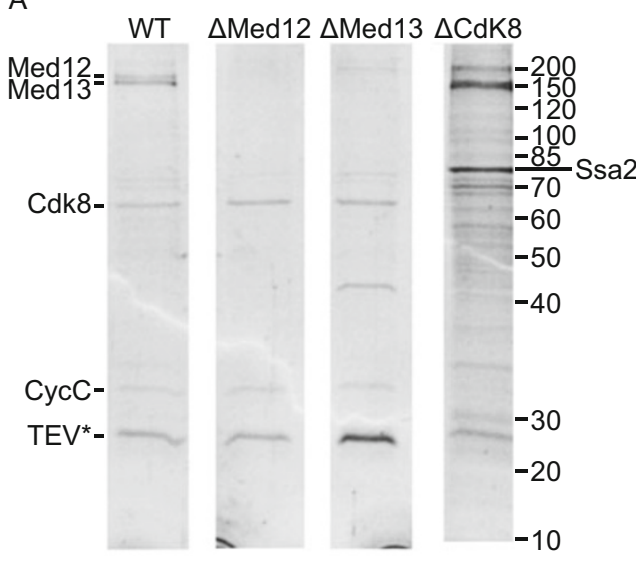

${ }^{B}$ Cdk8-CycC-Med12-Med13

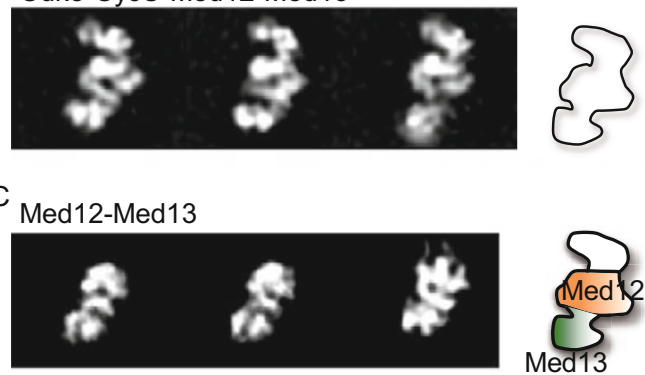

D Med12
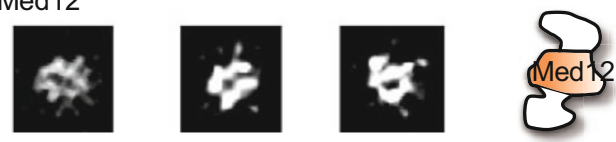

E

$$
\begin{array}{ll}
\text { Unlabeled } & \text { Labeled } \\
\text { averages } & \text { averages }
\end{array}
$$
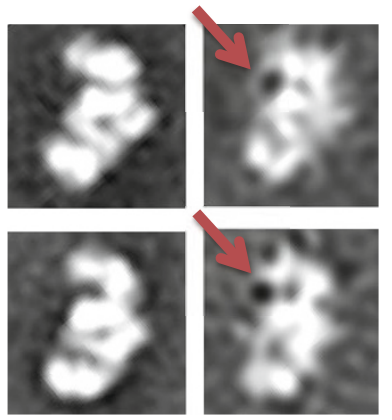

$\mathrm{F}$

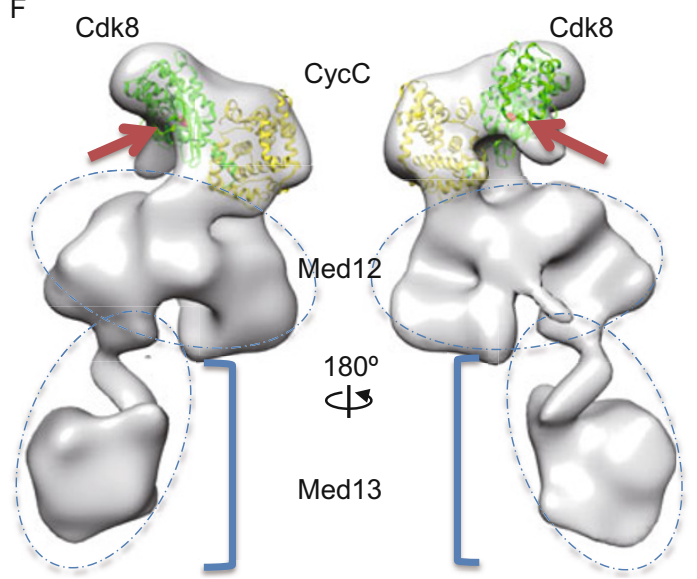

Figure 3. Cdk8 module subunit organization and EM characterization. (A) SDS-PAGE analysis of purified Cdk8 module and various deletions. Mass-spectrum analysis and Western blotting confirmed subunit composition. WT, wide type Cdk8 module preparation; $\Delta$ Med12, Med12 deletion mutant; $\Delta$ Med13, Med13 deletion mutant; $\Delta$ Cdk8, Cdk8 deletion mutant. Heat shock protein Ssa2 is co-purified with Cdk8 deletion mutant. (B) Two-dimensional class averages of wide type Cdk8 module including Cdk8, CycC, Med12, and Med13. (C) Representative 2D averages of the Med12-Med13 assembly purified from $\Delta$ Cdk8 mutant cells. (D) Representative 2D averages of Med12 subunit purified from cells with Med12-TAPm tagged yeast strain after extensive washing on IgG sepharose. Schematic representations: Cdk8-CycC (white), Med12 (orange), and Med13 (green). (E) Labeling of Cdk8 subunit with an Ni-NTA nanogold against the His 10 tag on C-terminal of Cdk8 results in gold cluster density highlighted by red arrowhead. (F) Unbiasedly docking the X-ray structure of the human Cdk8-CycC complex into the Cdk8 module 3D map and density corresponding to Med12 and Med13 subunits is denoted by blue dashed ellipses.

to the "hook" domain of the human Cdk8 module. The "hook" domain directly binds to human Mediator Tail module and corresponds to Med13 subunit. Therefore, the "Leg" domain of the yeast Cdk8 could probably be also functionally similar to the "hook" domain to mediate the Cdk8 module's interaction with core Mediator complex. This observation again substantiated that despite very low sequence homology, the structures of human and yeast Mediators are surprisingly similar.

\section{Subunit organization of the Cdk8 module}

In order to delineate the subunit organization of the Cdk8 module, we prepared multiple truncated forms of Cdk8 module through in vivo deletion of one subunit and analyzed the struc- tural difference attributable to the absence of the subunit to the structure of the full assembly consisting of all the four subunits. Using the same method to prepare the wide type Cdk8 module directly from yeast cells, we successfully purified truncated forms of Cdk8 module (Fig. 3A). The characterization of these complexes clearly shows deletion of either Med12 or Med13 could destabilize the whole Cdk8 module, leading to recovering of only Cdk8-CycC sub-complex. The result indicated that both Med12 and Med13 likely mediate the assembly and stabilization of the whole Cdk8 module.

When the corresponding class averages of the wide type, $\Delta$ Med12, $\Delta$ Med13, $\Delta$ Cdk8 were compared, a striking consistency and clear ordering was identified (Fig. 3B-D). In the 
averages of Med12-Med13 assembly purified from $\Delta$ Cdk8 mutant cells, the stable arm corresponding to Head portion of the Cdk8 module was entirely absent (Fig. 3C). The result suggested that the Head domain of Cdk8 module corresponds to Cdk8-CycC assembly. The 2D averages of Med12-Med13 sub-complex are much cleaner than these determined by Tsai et al. and help delineate the subunit assembly more clearly (Tsai et al., 2013). In addition, we have purified the Med12 subunit from cells with Med12-TAPm tagged yeast strain after extensive washing on IgG sepharose and EM analysis suggested Med12 subunit corresponds to the central density of the Cdk8 module in which both the two arms were missing (Fig. 3D).

Cdk8 subunit harbors the critical kinase activity and is also an important oncogene (Firestein et al., 2008; Adler et al., 2012). Localization of the Cdk8 subunit could not only provide structural insight into how Cdk8 kinase activity could be regulated by other subunits in the Cdk8 module, but also further identifies and validates the subunit organization determined using deletion mutant. We further validated the localization of Cdk8 subunit by nanogold labeling technique. An oligohistidine tag was incorporated on the C-terminus of Cdk8 and was targeted with Ni-NTA Nanogold particles (Nanoprobes). Class averages after incubation with nanogold clearly shows a small, well-defined area of high density close to Head domain of the Cdk8 module, which corresponded to gold cluster (Fig. 3E). The gold cluster labeling more precisely localized the C-terminus of the Cdk8 subunit to the tip of Head portion of the Cdk8 module structure. The localization of Cdk8 C-terminal is consistent with the result of localized $\mathrm{Cdk8}-\mathrm{CycC}$ assembly in the Head domain with the deletion mutants of Cdk8 module (Fig. $3 \mathrm{C}$ ). Moreover, the finding is further substantiated by unbiased docking the crystal structure of human Cdk8-CycC complex (PDB 3RGF) (Schneider et al., 2011) into the 3D structure of the module, which corresponded in size and shape (Fig. 3F).

Collectively, the results in Fig. 3 demonstrated that Med12 subunit forms the central lobe of the Cdk8 module (termed "Body"), while Med13 subunit forms the mobile "Leg" and Cdk8-CycC assembly corresponding to the "Head" portion (Fig. 3F). The Cdk8-CycC is located at the peripheral region of Cdk8 module and Med12 lines the walls of a central channel that may act as the platform for kinase catalysis. This interpretation provides a structural explanation for the regulation of Cdk8 kinase activity by Med12 observed in vivo and in vitro (Knuesel et al., 2009b). This structural arrangement suggested that Med12 could contribute to the tight regulation of Cdk8 kinase activity through regulating Cdk8 kinase's access to its phosphorylation substrates by modulating the structure of the central channel.

\section{Functional interaction of Cdk8 module with core Mediator in vivo}

Two previous publications on EM structural studies on Cdk8 module from fission yeast and human have reported it interacts differently with Mediator in the two species and could repress transcription through either occupying the RNAPII binding sites or locking the conformation of Mediator to prevent RNAPII binding, respectively (Elmlund et al., 2006; Knuesel et al., 2009a). The most recent publication on the EM characterization of S. cerevisiae Cdk8 module argued that it is a negative regulator and proposed a model that Cdk8 module inhibits transcription by blocking binding of RNA polymerase II to core Mediator (Tsai et al., 2013). According to this model, an interaction between core Mediator and the Cdk8 module should only result in transcription repression, which is contradictory with that Cdk8 module has been increasingly substantiated that could also contribute to transcription activation. Moreover, the model was based solely on EM image analysis and in vitro binding assays, and there was no single in vivo validation data presented. We could not exclude the possibility the model is oversimplified.

In order to achieve a comprehensive understanding of the Cdk8 module's regulation in both transcription activation and repression, we characterized, by structural and biochemical means both in vitro and in vivo, the interactions of Mediator with the RNAPII and Cdk8 module. To characterize the in vivo interaction of Cdk8 module with core Mediator, we performed co-immunoprecipitation (co-IP) experiment using the whole cell extraction of the S. cerevisiae with the Srb4/Med17 subunit tagged with Protein A. Both RNAPII and Cdk8 module were found to be co-precipitated with core Mediator. Interestingly, Mediator harbors strong interaction with RNAPII during transcription most inactive stationary phase (Fig. 4A). The finding is consistent with Mediator overlap with RNAPII on chromatin in stationary phase, which points to a role of Mediator as a binding platform for inactive Pol II during quiescence (Andrau et al., 2006). In contrast, Cdk8 module strongly interacts with core Mediator during exponential phase, when the transcription is highly active (Fig. 4A). The reciprocal co-IP experiment using the yeast cells with Cdk8 subunit tagged with Protein A also suggested Cdk8 module harbors strong interaction with core Mediator during transcription highly active exponential phase.

According to the model proposed by Tsai et al., Cdk8 module interaction with core Mediator will block RNAPII binding by binding to the presumed CTD binding site on the core mediator and interaction between core Mediator and the Cdk8 module could result in only transcription repression (Tsai et al., 2013). Our observation contradicts this model and suggests the model appears to be oversimplified. Using the co-IP assays at different growth conditions, we found the Cdk8 modulecore Mediator interaction is most prevalent during exponential phase when transcription activities are peaked, arguing against the model that interaction between the Cdk8 module with core Mediator could lead to only transcription repression (Tsai et al., 2013). Moreover, there is still substantial RNAPII bound to core Mediator when Cdk8 module-core Mediator interaction is most prevalent.

It has been suggested that Cdk8 module binds to the Mediator via the Med13 subunit from studies of human and 
A
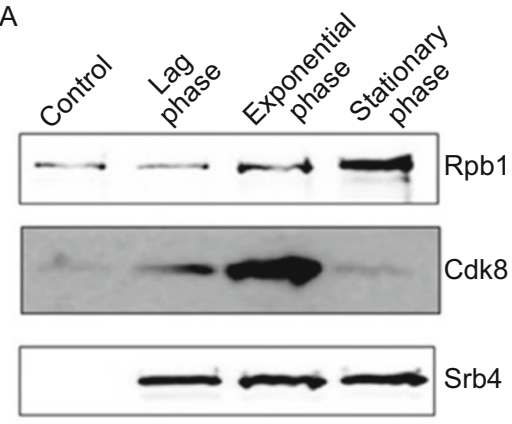

B

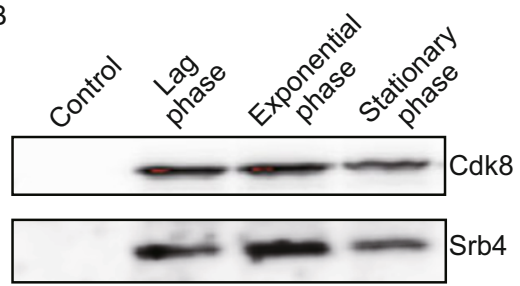

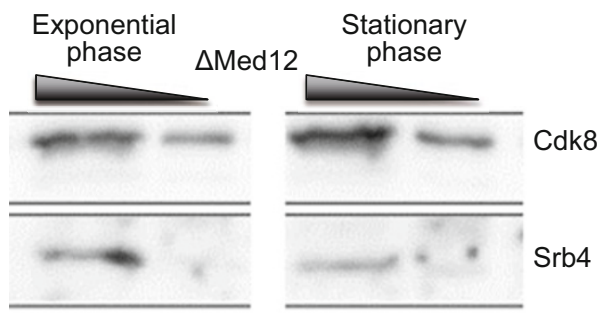

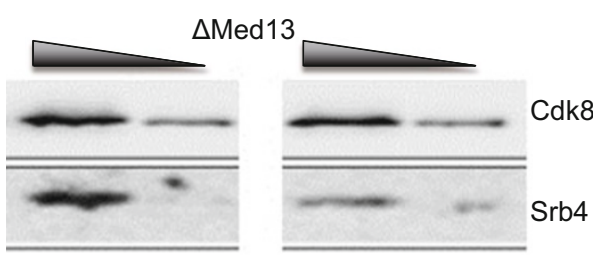

Figure 4. In vivo interaction of Cdk8 module with Mediator complex. (A) Co-immunoprecipitation of Cdk8::3HA with immunoprecipitated Srb4::Protein A. Protein crude extracts were prepared from strain WJY01 (Srb4::Protein A/Cdk8::3HA). Srb4::Protein A was immunoprecipitated by lgG sepharose. Proteins (IP) were separated by SDS-PAGE and revealed by Western blotting with monoclonal anti-CTD, mouse monoclonal anti-HA and PAP antibodies. The protein loading amount is normalized according to Srb4 content to compare the amount of Cdk8 and Rpb1 binding to Mediator in the yeast cells under different growth phases. (B) Co-immunoprecipitation of Srb4::3HA with immunoprecipitated Cdk8::Protein A. Protein crude extracts were prepared from strain WJY02 (Cdk8::Protein A / Srb4::3HA). Cdk8: :Protein A was immunoprecipitated by lgG sepharose. Proteins (IP) were separated by SDS-PAGE, and revealed by Western blotting with mouse monoclonal anti-HA and PAP antibodies. The protein-loading amount is normalized according to Cdk8 content to compare the amount of Mediator bound to Cdk8 module in the yeast cells of different growth phases. (C) Co-immunoprecipitation of Srb4 with immunoprecipitated Cdk8: :Protein A in the $\Delta$ Med12 (WJY03, Cdk8::Protein A/MMed12) and $\Delta$ Med13 cells (WJY04, Cdk8::Protein A / $\triangle$ Med13) during exponential and stationary phases. The yeast cells were immunoprecipitated by lgG sepharose and the Cdk8 loading amount is normalized to compare the amount of Mediator bound to the Cdk8 module.

S. pombe Cdk8 module (Elmlund et al., 2006; Knuesel et al., 2009a). Surprisingly, the co-immunoprecipitated S. cerevisiae core Mediator by Cdk8 module was not apparently reduced in $\Delta$ Med13, nor in $\Delta$ Med12 context (Fig. $4 \mathrm{C}$ ), unveiling Cdk8 module's interaction with Mediator does not depend on the Med12 nor Med13. This indicates that Cdk8 module may associate with the Mediator redundantly in vivo and that this association is, to a large extent, dependent on both Med13 and Med12. Another possibility is that Cdk8-CycC pair could take an active part in the interaction with core Mediator.

The data in Fig. 4B and $4 \mathrm{C}$ collectively suggested the persistent interactions of the Cdk8 module to core Mediator. Even in the case of Med12 or Med13 deletion, the rest of the Cdk8 module still stably interacts with core Mediator under all the growth conditions and the strong interaction of the Cdk8 module with core Mediator is concurrent with most active transcription states. Therefore, we propose Cdk8 module could contribute to transcription activation. Consistently, Cdk8 was found to be required during transcription elongation, where it is needed for recruitment of P-TEFb and BRD4 to transcribe RNAPII (Conaway and Conaway, 2011); Cdk8 is essential for some Diol transcription (Belakavadi and Fondell, 2010); and Cdk8 is a positive co-regulator of p53 target genes (Donner et al., 2007).
Functional interaction of Cdk8 module with core Mediator in vitro

As evidenced in our former publication, Mediator itself could harbor several different conformational states in solution (Fig. 5A). Single particle EM is ideally suited to characterize the conformations of Mediator under different conditions and indicate how it could be affected by interaction with various factors. Image alignment and classification can offer quantitative information about the effect of different factors on Mediator conformation. The critical information will advance our understanding of Mediator structural transitions, and to understand how those potential conformational changes of Mediator contribute to transcription and its regulation. We observed that incubation of core Mediator with RNAPII or Cdk8 module under the conditions normally used for structural characterization of core Mediator alone and found that both the RNAPII and Cdk8 module could dynamically bind to core Mediator independently of other factors.

Reference-free alignment and classification of core Mediator-RNAPII data suggested that RNAPII has alternative binding modes to core Mediator and could be tethered to the back and front side of Mediator (Fig. 5B). The RNAPII binding to back 


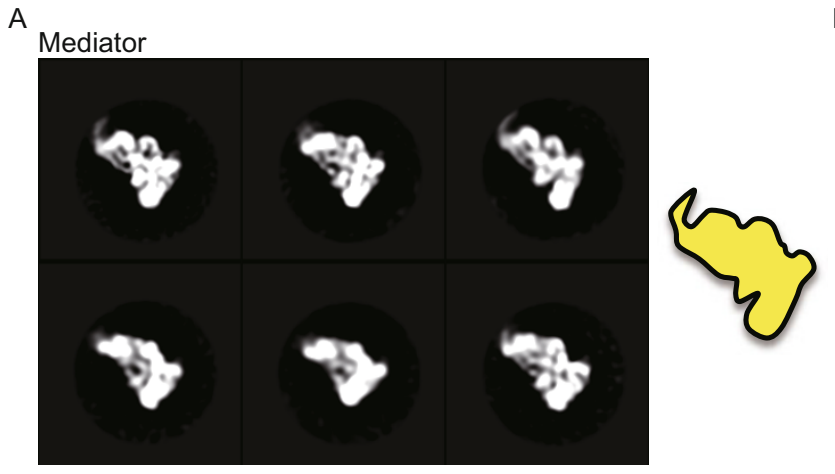

C
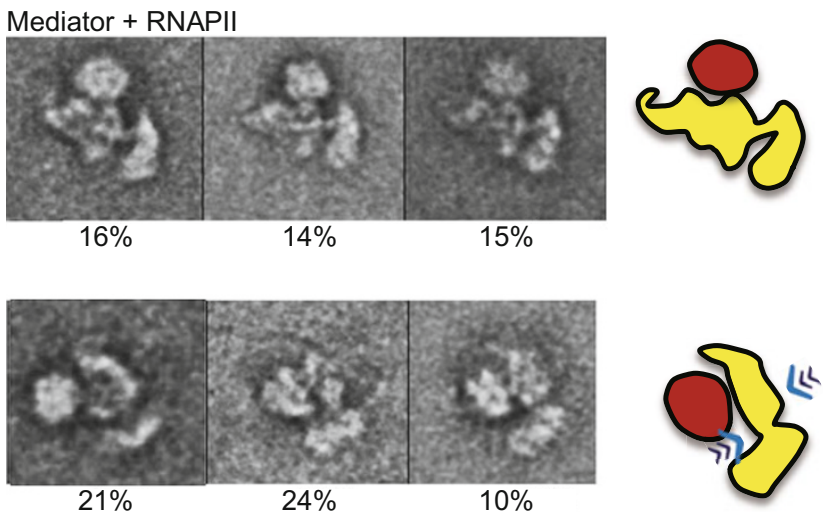
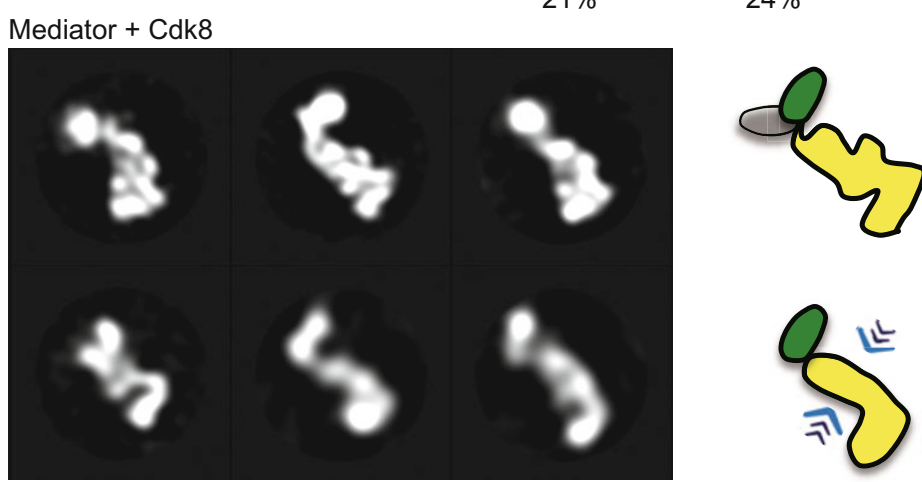

Figure 5. Functional interaction of Mediator with RNAPII and Cdk8 module. (A) Class averages of Mediator obtained after referencefree alignment of the images of Mediator complex preserved in stain (left). Schematic representation: Mediator of typical conformation (right). (B) Class averages obtained after reference-free alignment of the images of core Mediator-RNAPII assembly (left). Schematic representation of RNAPII tethered to the back and front side of core Mediator. The blue arrows highlight the conformational changes of Mediator upon binding to RNAPII (right). (C) Class averages obtained after reference-free alignment of the images in the core Mediator-Cdk8 data set showing direct interactions. Cdk8 module corresponded to the localized density adjacent to the Tail module of core Mediator (left). Schematic representation of core Mediator bound by Cdk8 module. The blue arrows highlight the substantial conformational changes of core Mediator into open conformation upon binding to Cdk8 module (right).

side of Mediator represents the predominant interaction mode, which constitute about $70 \%$ of the RNAPII binding to core Mediator (Fig. 5B, top panel). The core Mediator adopts the "closed" conformation, which shows no difference with that of the core Mediator alone. Only about $30 \%$ of the RNAPII binding on the front side of Mediator (Fig. 5B, bottom panel), which appears to embrace RNAPII to form so-called holoenzyme (Davis et al., 2002). Interestingly, core Mediator displayed a partially extended "open" conformation resulting in dissociation of the Tail and Middle module. It is tempting to speculate the core Mediator in the partially "open" conformation could represent an active state of Mediator. If this is the case, the majority core Mediator is in the "closed" conformation and probably in the inactive state. This is consistent with the data in Fig. 4A, showing that in stationary phase, core Mediator-RNAPII interaction appears to be predominant, which is concurrent with transcription inactivation. These observations are also consistent with Mediator overlap with RNAPII on chromatin in stationary phase, which points to a role of Mediator as a binding platform for inactive RNAPII during quiescence (Andrau et al., 2006).
Therefore, we propose core Mediator-RNAPII interaction could not directly correlate to transcription activation, instead majority of such interactions represent transcription inactive states.

Detailed image analysis of the core Mediator-Cdk8 module dataset suggested that core Mediator was induced into the wide "open" conformation upon binding with Cdk8 module. The conformational changes of core Mediator are so substantial that resulting in wide dissociation of the Middle and Tail module of core Mediator (Figs. 5C and S2). Interestingly, the mode of interaction between core Mediator and the Cdk8 module is strikingly similar to that of human, in which Cdk8 module is tethered to the tip of Tail module of core Mediator, and different from the finding of Tsai et al., in which the Middle module of core Mediator was suggested to bind Cdk8 module (Tsai et al., 2013). Most importantly, an interaction with the Cdk8 module induces core Mediator to adopt the wide "open" conformation (Fig. 5C, bottom panel).

Since a major change in Mediator conformation is essential for generating a surface for assembly of the pre-initiation complex (PIC), structural transition of Mediator should constitute 
a critical regulation point for eukaryotic transcription (Chadick and Asturias, 2005). The "open" conformation of core Mediator is induced by interaction with Cdk8 module, suggesting the possibility that a positive role of the Cdk8 module is to induce core Mediator open, which is presumed to be an active state of Mediator. Consistently, the Cdk8 module interaction with Mediator in vivo is concurrent with active transcription, suggesting its involvement in transcription activation. These observations suggested that Cdk8 module could regulate transcription positively by inducing core Mediator into open conformation. It will thus be interesting to investigate how Cdk8 module could critically contribute to transcription regulation both positively and negatively by modulating Mediator conformation accordingly.

In summary, we reported a substantially improved 3D EM reconstruction of Mediator Cdk8 module in several conformations, which showed considerably more structural details and provides a new level of understanding of Cdk8 module structural organization. The elucidation of subunit organization and inherent structural flexibility critically contributes to an understanding of a possible mechanism by which Med12 could contribute to the tight regulation of Cdk8 kinase activity through regulating Cdk8 subunit's access to its phosphorylation substrates. The Cdk8 module's interaction with core Mediator in vivo is concurrent with active transcription, suggesting its involvement in transcription activation. An interaction with the Cdk8 module induces core Mediator into "open" conformation. These suggested Cdk8 module could possibly regulate transcription by modulating Mediator conformation. Taken together, the data presented here provide novel insights into the molecular mechanism of Cdk8 module for transcriptional regulation, in addition to a detailed picture of the protein interactions within this complex.

\section{MATERIALS AND METHODS}

Cdk8 module biochemical purification

A modified TAP (10x histidine-TEV-Protein A) tag was fused to the Cterminus of the Cdk8 or Med12 subunit, using a PCR-based genomic epitope-tagging method in the multiple protease-deficient yeast strain BJ2168 (MATa leu2 trp1 ura3-52 prb1-1122 pep4-3 prc1-407 gal2). The affinity purification procedure is the same with our published protocol for Mediator preparation, with minor modifications (Cai et al., 2009). The purity and components of the Cdk8 module were confirmed by SDSPAGE, followed by silver staining or Western blotting. To further improve the purity and homogeneity, the elution from the $\lg G$ column was applied onto a $1 \mathrm{~mL}$ Mono $\mathrm{Q}$ column (GE Healthcare) in Q100 buffer

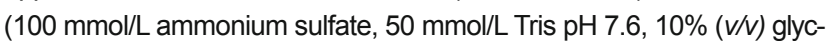
erol, $1 \mathrm{mmol} / \mathrm{L}$ EDTA, $10 \mu \mathrm{mol} / \mathrm{L} \mathrm{ZnSO}{ }_{4}, 0.02 \%$ NP-40, $10 \mathrm{mmol} / \mathrm{L} \beta-\mathrm{ME}$ ) and was resolved over a $100-1000 \mathrm{mmol} / \mathrm{L}$ ammonium sulfate gradient. The final Cdk8 module fractions eluted at $\sim 300 \mathrm{mmol} / \mathrm{L}$ ammonium sulfate concentration were flash-frozen in liquid nitrogen.

\section{Electron microscopy sample preparation and data collection}

About $3 \mu \mathrm{L}$ Cdk8 module aliquots $(\sim 20 \mu \mathrm{g}$ protein $/ \mathrm{mL}$ in $50 \mathrm{mmol} / \mathrm{L}$ Tris $\mathrm{pH} 7.6,100 \mathrm{mmol} / \mathrm{L}$ ammonium sulfate, $10 \%(\mathrm{v} / \mathrm{v})$ glycerol, $1 \mathrm{mmol} / \mathrm{L}$
EDTA, $10 \mu \mathrm{mol} / \mathrm{L} \mathrm{ZnSO}_{4}, 0.02 \% \mathrm{NP}-40,10 \mathrm{mmol} / \mathrm{L} \beta-\mathrm{ME}$ ) was applied to a carbon-coated 400-mesh Cu EM specimen grid freshly glow discharged. Cdk8 module particles were then preserved by staining with $0.75 \%(w / w)$ uranyl formate solution.

We obtained tilted $\left(-55^{\circ}\right)$ and untilted image pairs of Cdk8 module particles under low-dose conditions using a Tecnai F20 microscope (FEI) (field emission gun, $200 \mathrm{kV}$ accelerating voltage, $\sim 0.6-0.8 \mu \mathrm{m}$ underfocus). Images were recorded with a FEI Eagle (4k HS 200 kV) CCD camera at $62,000 \times$ magnification (1.77 $\AA$ per pixel) and two-fold pixel-averaged to $3.54 \AA$ per pixel. Particles were selected using the TiltPicker program (Voss, et al., 2009) and montaged them for interactive screening, yielding $~ 9000$ single particle tilt-pair images.

\section{Cdk8 subunit labeling}

Gold cluster labeling of Cdk8 module was carried out by incubating Cdk8 module at $20 \mu \mathrm{g}$ protein $/ \mathrm{mL}$ with a 10 -fold molar excess of Ni-NTA (Nanoprobe) for $2 \mathrm{~h}$ at $4^{\circ} \mathrm{C}$, then dialyzed against the buffer (50 mmol/L Tris, $\mathrm{pH} \mathrm{7.6,100} \mathrm{mmol/L} \mathrm{ammonium} \mathrm{sulfate,} 1 \mathrm{mmol} / \mathrm{L}$ EDTA, $10 \mu \mathrm{mol} / \mathrm{L} \mathrm{ZnSO} 4,0.01 \% \mathrm{NP}-40$ ) for another $2 \mathrm{~h}$ at $4^{\circ} \mathrm{C}$. EM samples were prepared and imaged as described above.

\section{Yeast strains constructions}

For subunit truncation of Med12, Med13, and Cdk8, a PCR-amplified kanMX6 cassette was used to replace the open reading frame of specific subunits in the background of BJ2168 resulting in construction of strains of $\Delta$ Med12, $\Delta$ Med13, and $\Delta$ Cdk8 mutants. WJY01 (Srb4: : Protein A/Cdk8: :3HA) is constructed by fusing a modified (10× histidine-TEV-Protein A) tag to the C-terminus of the Med17/Srb4 subunit and three copies of the HA epitope into the C-terminus of the Cdk8 subunit. WJY02 (Cdk8::Protein A/Srb4::3HA) is constructed by fusing a modified (10× histidine-TEV-Protein $A)$ tag to the C-terminus of the Cdk8 subunit and three copies of the HA epitope into the C-terminus of the Med17/Srb4 subunit. WJY03 (Cdk8::Protein A/ $\Delta$ Med12) and WJY04 (Cdk8: :Protein A/DMed13) is constructed by inserting a modified (10× histidine-TEV-Protein A) tag to the C-terminus of the Cdk8 subunit in the background of $\Delta$ Med12, $\Delta$ Med13 strains, respectively.

\section{Three-dimensional reconstruction}

We calculated $3 \mathrm{D}$ reconstructions using the random conical tilt (RCT) method (Radermacher, 1988). We initially analyzed 2D images using the Xmipp and Sparx package (Hohn et al., 2007; Knuesel et al., 2009b). We then used the resulting averages to run iterative alternating rounds of supervised multi-reference alignment and classification as well as reference-free alignment with Spider to improve the homogeneity of the image classes (Frank et al., 1996, Cai et al., 2010). We produced all molecular graphics images using Chimera (Pettersen et al., 2004).

\section{EM analysis of Mediator-RNAPII and Mediator-Cdk8 module interaction}

The assembly of Mediator-RNAPII and Mediator-Cdk8 module was assembled by incubation Mediator with a five-fold molar excess of RNAPII and Cdk8 module for $4 \mathrm{~h}$ at $4^{\circ} \mathrm{C}$. The resulting complexes were negatively stained and examined by EM as described above. 


\section{ACKNOWLEDGMENTS}

This work was supported by grants from the National Basic Research Program (973 Program) (No. 2013CB910200), the National Natural Science Foundation of China (Grant Nos. 31170694 and 31222017), Ministry of Education of PRC (NCET-11-0874) and Chinese Academy Of Sciences (48500108-6).

\section{ABBREVIATIONS}

RCT, random conical tilt; RNAPII, RNA polymerase II; WT, wide type Cdk8 module; $\Delta$ Cdk8, Cdk8 deletion mutant; $\Delta$ Med12, Med12 deletion mutant; $\Delta$ Med13, Med13 deletion mutant

\section{COMPLIANCE WITH ETHICS GUIDELINES}

Xuejuan Wang, Jianye Wang, Zhenrui Ding, Jinhua Ji, Qianqian Sun and Gang Cai declare that they have no conflict of interest.

This article does not contain any studies with human or animal subjects performed by the any of the authors.

\section{REFERENCES}

Adler, A.S., McCleland, M.L., Truong, T., Lau, S., Modrusan, Z., Soukup, T.M., Roose-Girma, M., Blackwood, E.M., and Firestein, R. (2012). CDK8 maintains tumor dedifferentiation and embryonic stem cell pluripotency. Cancer Res 72, 2129-2139.

Andrau, J.C., van de Pasch, L., Lijnzaad, P., Bijma, T., Koerkamp, M.G., van de Peppel, J., Werner, M., and Holstege, F.C. (2006). Genomewide location of the coactivator mediator: Binding without activation and transient Cdk8 interaction on DNA. Mol Cell 22, 179-192.

Asturias, F.J., Jiang, Y.W., Myers, L.C., Gustafsson, C.M., and Kornberg, R.D. (1999). Conserved structures of mediator and RNA polymerase II holoenzyme. Science 283, 985-987.

Belakavadi, M., and Fondell, J.D. (2010). Cyclin-dependent kinase 8 positively cooperates with Mediator to promote thyroid hormone receptor-dependent transcriptional activation. Mol Cell Biol 30, 2437-2448.

Bernecky, C., Grob, P., Ebmeier, C.C., Nogales, E., and Taatjes, D.J. (2011). Molecular architecture of the human Mediator-RNA polymerase II-TFIIF assembly. PLoS Biol 9, e1000603.

Borggrefe, T., Davis, R., Erdjument-Bromage, H., Tempst, P., and Kornberg, R.D. (2002). A complex of the Srb8,-9,-10, and-11 transcriptional regulatory proteins from yeast. J Biol Chem 277, 44202-44207.

Cai, G., Chaban, Y.L., Imasaki, T., Kovacs, J.A., Calero, G., Penczek, P.A., Takagi, Y., and Asturias, F.J. (2012). Interaction of the mediator head module with RNA polymerase II. Structure 20, 899-910.

Cai, G., Imasaki, T., Takagi, Y., and Asturias, F.J. (2009). Mediator structural conservation and implications for the regulation mechanism. Structure 17, 559-567.

Cai, G., Imasaki, T., Yamada, K., Cardelli, F., Takagi, Y., and Asturias, F.J. (2010). Mediator head module structure and functional interactions. Nat Struct Mol Biol 17, 273-279.

Chadick, J.Z., and Asturias, F.J. (2005). Structure of eukaryotic Mediator complexes. Trends Biochem Sci 30, 264-271.

Conaway, R.C., and Conaway, J.W. (2011). Function and regulation of the Mediator complex. Curr Opin Genet Dev 21, 225-230.

Davis, J.A., Takagi, Y., Kornberg, R.D., and Asturias, F.A. (2002). Structure of the yeast RNA polymerase II holoenzyme: Mediator conformation and polymerase interaction. Mol Cell 10, 409-415.

Donner, A.J., Ebmeier, C.C., Taatjes, D.J., and Espinosa, J.M. (2010). CDK8 is a positive regulator of transcriptional elongation within the serum response network. Nat Struct Mol Biol 17, 194-201.

Donner, A.J., Szostek, S., Hoover, J.M., and Espinosa, J.M. (2007). CDK8 is a stimulus-specific positive coregulator of p53 target genes. Mol Cell 27, 121-133.

Dotson, M.R., Yuan, C.X., Roeder, R.G., Myers, L.C., Gustafsson, C.M., Jiang, Y.W., Li, Y., Kornberg, R.D., and Asturias, F.J. (2000). Structural organization of yeast and mammalian mediator complexes. Proc Natl Acad Sci U S A 97, 14307-14310.

Elmlund, H., Baraznenok, V., Lindahl, M., Samuelsen, C.O., Koeck, P.J., Holmberg, S., Hebert, H., and Gustafsson, C.M. (2006). The cyclin-dependent kinase 8 module sterically blocks Mediator interactions with RNA polymerase II. Proc Natl Acad Sci U S A 103, 15788-15793.

Firestein, R., Bass, A.J., Kim, S.Y., Dunn, I.F., Silver, S.J., Guney, I., Freed, E., Ligon, A.H., Vena, N., Ogino, S., et al. (2008). CDK8 is a colorectal cancer oncogene that regulates beta-catenin activity. Nature 455, 547-551.

Frank, J., Radermacher, M., Penczek, P., Zhu, J., Li, Y., Ladjadj, M., and Leith, A. (1996). SPIDER and WEB: processing and visualization of images in 3D electron microscopy and related fields. J Struct Biol 116, 190-199.

Hohn, M., Tang, G., Goodyear, G., Baldwin, P.R., Huang, Z., Penczek, P.A., Yang, C., Glaeser, R.M., Adams, P.D., and Ludtke, S.J. (2007). SPARX, a new environment for Cryo-EM image processing. J Struct Biol 157, 47-55.

Holstege, F.C., Jennings, E.G., Wyrick, J.J., Lee, T.I., Hengartner, C.J., Green, M.R., Golub, T.R., Lander, E.S., and Young, R.A. (1998). Dissecting the regulatory circuitry of a eukaryotic genome. Cell 95, 717-728.

Knuesel, M.T., Meyer, K.D., Bernecky, C., and Taatjes, D.J. (2009a). The human CDK8 subcomplex is a molecular switch that controls Mediator coactivator function. Genes Dev 23, 439-451.

Knuesel, M.T., Meyer, K.D., Donner, A.J., Espinosa, J.M., and Taatjes, D.J. (2009b). The human CDK8 subcomplex is a histone kinase that requires Med12 for activity and can function independently of mediator. Mol Cell Biol 29, 650-661.

Kornberg, R.D. (2005). Mediator and the mechanism of transcriptional activation. Trends Biochem Sci 30, 235-239.

Lariviere, L., Seizl, M., and Cramer, P. (2012). A structural perspective on Mediator function. Curr Opin Cell Biol 24, 305-313.

Lee, J.H., Cai, G., Panigrahi, A.K., Dunham-Ems, S., Nguyen, T.N., Radolf, J.D., Asturias, F.J., and Gunzl, A. (2010). A TFIIH-associated mediator head is a basal factor of small nuclear spliced leader RNA gene transcription in early-diverged trypanosomes. Mol Cell Biol 30, 5502-5513.

Lee, T.I., and Young, R.A. (2000). Transcription of eukaryotic proteincoding genes. Annu Rev Genet 34, 77-137.

Malik, S., and Roeder, R.G. (2000). Transcriptional regulation through Mediator-like coactivators in yeast and metazoan cells. Trends Biochem Sci 25, 277-283.

Myers, L.C., Gustafsson, C.M., Bushnell, D.A., Lui, M., ErdjumentBromage, H., Tempst, P., and Kornberg, R.D. (1998). The Med proteins of yeast and their function through the RNA polymerase II carboxy-terminal domain. Genes Dev 12, 45-54. 
Naar, A.M., Lemon, B.D., and Tjian, R. (2001). Transcriptional coactivator complexes. Annu Rev Biochem 70, 475-501.

Pettersen, E.F., Goddard, T.D., Huang, C.C., Couch, G.S., Greenblatt, D.M., Meng, E.C., and Ferrin, T.E. (2004). UCSF Chimera--a visualization system for exploratory research and analysis. J Comput Chem 25, 1605-1612.

Radermacher, M. (1988). Three-dimensional reconstruction of single particles from random and nonrandom tilt series. J Electron Microsc Tech 9, 359-394.

Schneider, E.V., Bottcher, J., Blaesse, M., Neumann, L., Huber, R., and Maskos, K. (2011). The structure of CDK8/CycC implicates specificity in the CDK/cyclin family and reveals interaction with a deep pocket binder. J Mol Biol 412, 251-266.

Taatjes, D.J., Naar, A.M., Andel, F., Nogales, E., and Tjian, R. (2002). Structure, function, and activator-induced conformations of the CRSP coactivator. Science 295, 1058-1062.

Tsai, K.L., Sato, S., Tomomori-Sato, C., Conaway, R.C., Conaway, J.W., and Asturias, F.J. (2013). A conserved Mediator-CDK8 kinase module association regulates Mediator-RNA polymerase II interaction. Nat Struct Mol Biol 20, 611-619.

Voss, N.R., Lyumkis, D., Cheng, A., Lau, P.W., Mulder, A., Lander, G.C., Brignole, E.J., Fellmann, D., Irving, C., Jacovetty, E.L., et al. (2010). A toolbox for ab initio 3-D reconstructions in single-particle electron microscopy. J Struct Biol 169, 389-398. 